\title{
Deep-UV Remote Raman Detection of Chlorine
}

\author{
Emanuela Gallo ${ }^{1}$, Frank Duschek ${ }^{1}$ \\ ${ }^{1}$ German Aerospace Center, Institute of Technical Physics, Langer Grund 1, 74239 Hardthausen, Germany \\ emanuela.gallo@dlr.de
}

\begin{abstract}
Deep ultraviolet Raman spectroscopy has been performed to detect chlorine gas in a remote configuration. Several laser wavelengths were employed to observe the optimum signal-tobackground ratio. Detection limits in acquisition times are discussed.
\end{abstract}

A short range remote backscattering Raman set up was developed to measure chlorine gas for a possible first alert and monitoring through the application of a Raman scattering based detector. Unwanted chlorine release into the atmosphere can occur as accidental industrial spill, domestic exposure, and warfare agent [1]. High concentrations (400 ppm fatal over 30 minutes, $>1000$ ppm mortality in few minutes [2]) of this yellow-green pale gas can cause death by asphyxiation. The development of a system capable of monitoring and detecting reasonably fast (few seconds of acquisition time), identifying the unknown compound, and ideally sampling remotely (without getting the personnel in contact with the possible danger) is necessary [3,4]. For these reasons, a remote configuration was implemented due to its capability of avoiding direct contact with the source of unknown danger [5]. Out of all the possibilities [6] (infrared absorption, for example, cannot detect symmetric molecules like chlorine gas), Raman spectroscopy is capable to uniquely identify an unknown substance. Diatomic molecules like chlorine are Raman active and the Raman signal increases drastically lowering the laser wavelength [6]. Therefore, laser excitation wavelengths in the ultraviolet (UV) region were chosen. Tests were conducted using a UV dye laser to generate tunable excitation wavelengths and a spectrometer coupled with a nitrogen cooled charged coupled device (CCD) as detector. UV Raman spectra of chlorine were detected over a remote distance of 60 centimeters (laser energy density below $20 \mathrm{~mJ} / \mathrm{cm}^{2}$ ). Several UV laser wavelengths $(224,232,235 \mathrm{~nm})$ were applied to experimentally observe and maximize the Raman signal. For each tested excitation wavelength, chlorine spectra were successfully detected. Detection limits given in acquisition time are discussed. When performing a test in a closed laboratory environment any possible chlorine release has to be precluded. Hence the chlorine must be enclosed into a sealed cell which introduces a potential additional source for a Raman signal, which would be absent in a realistic scenario. Discriminating the acquired sample signal from the signature of the cell material was challenging since both Raman spectral lines overlapped [7]. In this paper a backscattering configuration will be described and a solution to discriminate the contribution of the sampled signal from the interfering background will be presented. Fig. 1a represents the sample (chlorine and residual air) signal overlapping with the unwanted Raman signal of a commercial Suprasil-qs quartz cell (thickness of probed gas volume of $10 \mathrm{~mm}$ at chlorine concentration of $40 \% \mathrm{vol}$ ) Fig. 1b was acquired changing the cell material to a Crystran Raman grade calcium fluoride $\left(\mathrm{CaF}_{2}\right)$ [8] to avoid spectral overlapping regions around chlorine. Chlorine signal can be clearly distinguished as a sharp peak at $554 \mathrm{~cm}^{-}$ ${ }^{1}$ [9] while residual air peaks are located at $1556 \mathrm{~cm}^{-1}$ for atmospheric oxygen and $2331 \mathrm{~cm}^{-1}$ for atmospheric nitrogen [6].

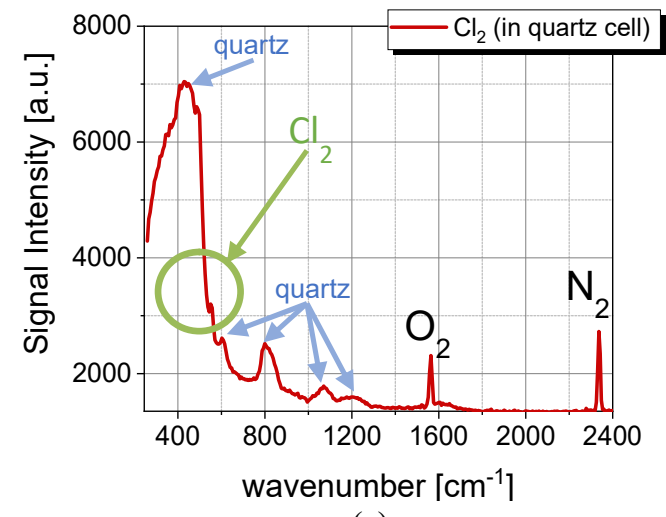

(a)

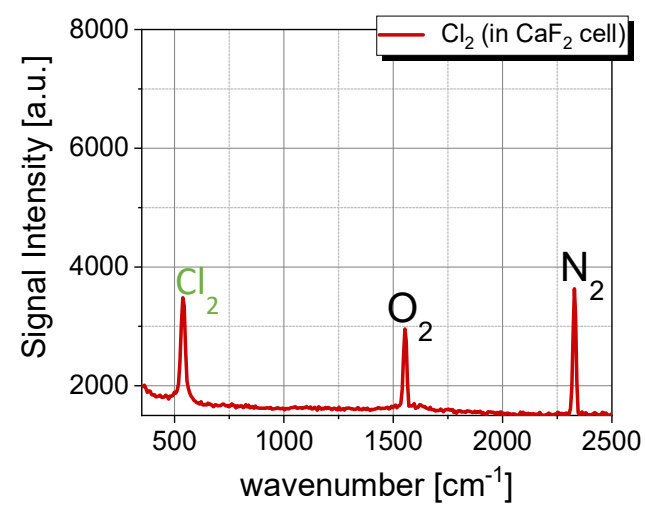

(b)

Fig 1. (a) Quartz and chlorine Raman raw signal, (b) $\mathrm{CaF}_{2}$ and chlorine Raman raw signal 
Fig. 2a represents the signal-to-background ratio of chlorine gas while changing laser wavelengths still in the quartz glass cell showing a maximum for $235 \mathrm{~nm}$. The time detection limit was limited to $50 \mathrm{~s}$ (red line) due to the cell material interference. Fig $2 \mathrm{~b}$ shows experiments performed at $235 \mathrm{~nm}$ using a Raman grade $\mathrm{CaF}_{2}$ cell. The acquisition time was lowered by an order of magnitude compared to the quartz cell $(5 \mathrm{~s})$.

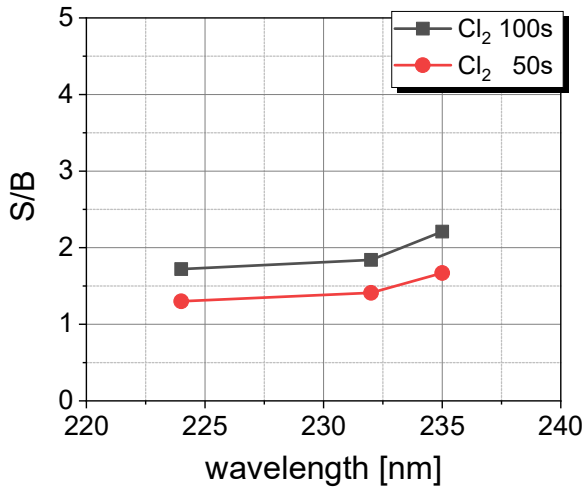

(a)

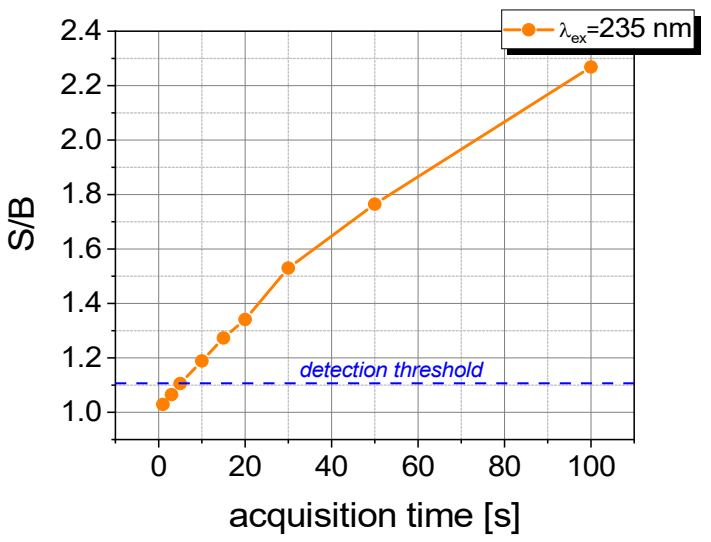

(b)

Fig. 2 Signal-to-background ratio of $\mathrm{Cl}_{2}$ (a) vs wavelength, 100s (black line) and 50s (red line) sampling time - quartz cell and (b) vs acquisition time $\mathrm{CaF}_{2}$ cell (laser at 235nm)

Within this study, it was possible to enhance the quality of the chlorine Raman signal resulting in improved detection times of 5 seconds of acquisition time overcoming the spectral overlapping from a gas cell material initially founded. Additional laser excitation wavelengths will be presented to find the optimal excitation wavelength which maximizes the signal of chlorine gas. A later system improvement would rely on a featuring gated sensor like intensified coupled charged device (iCCD) camera and/or a stack of photo multiplier tubes (PMT) to further lower the detection limits by an expected additional order of magnitude.

\section{References}

[1] C. Winder, "The Toxicology of Chlorine”, Vol. 85, Issue 2 (Elsevier Environmental Research, 2001), pp. 105-114

[2] C. W. White, J. G. Martin, Vol. 7 Issue 4, "Chlorine gas inhalation: human clinical evidence of toxicity and experience in animal models", (Proceedings of the American Thoracic Society, 2010), pp 257-263

[3] L.A. Skvortsov, "Laser Methods for Detecting Explosive Residues on Surfaces of Distant Objects", Vol. 16, (Quantum Electronics, 2012), pp. 8

[4] R. L. Aggarwal, L. W. Farrar, S. Di Cecca, M. Amin, B. G. Perkins, M. L. Clark, T. H. Jeys, D. W. Sickenberger, F. M. D’Amico, E. D. Emmons, S. D. Christesen, R. J. Kreis, G. K. Kilper, "Chemical aerosol Raman detector”, Vol 88, Issue 3, (AIP Review of Scientific Instruments, 2017)

[5] E. Gallo, C. Kölbl, F. Duschek, A. Köhntopp, A. Walter, "UV Raman Spectroscopy for Explosives", (3rd International Conference CBRNE- Research \& Innovation, Nantes, France, 2019)

[6] A.C. Eckbreth, "Laser diagnostics for combustion temperature and species", Vol.3, $2^{\text {nd }}$ ed., (Combustion Science and Technology, 1996), Gordon and Breach publisher

[7] L. Skuja, K. Kajihara, K. Smits, A. Silinis, H. Hosono, "Luminescence and Raman Detection of Molecular Cl2 and ClClO Molecules in Amorphous SiO2 Matrix", Vol. 121, Issue 9, (Journal of Physical Chemistry, 2017), pp 5261-5266

[8] Crystran Raman grade calcium fluoride material properties: https://www.crystran.co.uk/raman-substrate-materials

[9] R.L. Aggarwal, L.W. Farrar, S. Di Cecca, T.H. Jeys, "Raman spectra and cross sections of ammonia, chlorine, hydrogen sulfide, phosgene, and sulfur dioxide toxic gases in the fingerprint region 400-1400 cm-1", Vol.6, Issue 2, (AIP Advances, 2016) 